\title{
Mechanical response of oxidized Zircaloy-4 cladding material submitted to a ring compression test
}

\author{
Vincent Busser ${ }^{\mathrm{a}, *}$, Marie-Christine Baietto-Dubourg ${ }^{\mathrm{b}}$, Jean Desquines ${ }^{\mathrm{a}}$, Christian Duriez ${ }^{\mathrm{a}}$, \\ Jean-Paul Mardon ${ }^{c}$ \\ a IRSN, DPAM, 13115, Saint-Paul-lez-Durance cedex, France \\ ${ }^{\mathrm{b}}$ LaMCoS, Insa-Lyon, CNRS, UMR 5259, F-69621, France \\ ' AREVA NP SAS, 10 rue Juliette, Récamier, 69456 Lyon cedex 06, France
}

The ring compression test method has been evaluated as a tool to assess Zircaloy- 4 cladding mechanical properties and to analyze oxide layer damage evolution with applied strain. For sufficient axial length, the test is shown to develop plane strain conditions at the most deformed location. The test analysis requires finite element calculations. The aim of the paper is to highlight the potentialities of the ring compression test method.

\section{Introduction}

Zircaloy- 4 is one of the most used fuel-cladding alloys in pressurized water reactor (PWR). The PWR primary water oxidizes the cladding during irradiation. Thus, a zirconia layer develops at the outer surface of the Zircaloy-4 cladding up to a layer thickness of about $100 \mu \mathrm{m}$ for high burnup fuel rods.

In the frame of its research activities on nuclear fuel safety, the French 'Institut de Radioprotection et de Sûreté nucléaire' tested irradiated PWR fuel rods in the CABRI facility. The CABRI REP-Na program was launched in 1991 to study the consequences of a reactivity initiated accident (RIA) for high burnup fuel rods. This program evidenced some important phenomena. One of them was zirconia cracking and spallation during the RIA transient. Parallel cracks, induced by plastic deformation, formed a network of zirconia fragments. The average oxide fragment size decreases with increasing strain, and then saturates at high strain levels [1]. These fragments spalled for higher strain levels. Oxide thickness has a major influence on the cracking and spallation mechanisms. The zirconia layer acts as a very efficient thermal insulator and has a major influence on the thermal-mechanical behavior of the fuel rod. Oxide spallation modifies significantly the temperature distribution in the cladding. It is thus important to better characterize the mechanical conditions at which oxide spallation occurs. The ring compression test (RCT) appears to be an interesting tool to address this topic with a limited amount of testing.

* Corresponding author. Tel.: +33 442199490; fax: +33 442199166 .

E-mail addresses: vincent.busser@irsn.fr (V. Busser), marie-christine.baietto@ insa-lyon.fr (M.-C. Baietto-Dubourg), jean.desquines@irsn.fr (J. Desquines), christian.duriez@irsn.fr (C. Duriez), jeanpaul.mardon@areva.com (J.-P. Mardon).
Some investigations were carried out on aluminum ring compression test [2-4], which focused on predicting either hoop stress or strain curve for tube materials. Authors introduced analytical models providing determination of the yield stress and the fracture strain. However, identifying the mechanical properties by an analytical approach appeared to have limited accuracy. Lateral compression of aluminum tube was recently studied with a finite element method (FEM) approach and it has been proven that it is possible to deduce the mechanical properties of the material by inverse modeling [5]. FEM calculation also shows that the maximum strain obtained by RCT can reach high values (more than $40 \%$ in the case of aluminum ring). Moreover, strain evolution versus angular position was measured and it has also been shown that strain induced by RCT is localized at the equatorial azimuth of the ring [6]. The large range of strain values along the ring circumference has also been highlighted on ring compression test performed on composite materials [7].

For loss of coolant accident (LOCA) studies, ring compression tests on cladding tubes are usually performed on high temperature oxidized and quenched material to check the material embrittlement [8]. In this context, the considered material is highly heterogeneous, involving a stratified microstructure. The test result is rather an empirical evaluation of the cladding residual ductility. The modeling of this test is thus complex but attempts to perform finite element modeling provided recently interesting results [9].

Because of its ability to generate high strain levels with steep gradients along the specimen, lateral ring compression test (RCT) on Zircaloy-4 cladding tube contributes to analyze the influence of a large range of strain levels on the oxide cracking and spallation mechanisms. This paper will focus more specifically on the mechanical behavior of oxidized rings submitted to RCT. The 
analysis of the oxide degradation as a function of strain will be briefly introduced but it will not be fully addressed.

\section{Experimental procedure}

\subsection{Material and oxidation}

Samples are cladding tube segments constituted of low tin Zircaloy-4 (Zy-4) supplied by Areva NP. Two different metallurgical states, resulting from two final heat treatments, have been investigated: recrystallized annealed (RXA) and stress-relieved annealed (SRA) Zircaloy-4. Chemical compositions of the alloys are given in Table 1. Outer diameter and wall thickness of the cladding are, respectively, $9.5 \mathrm{~mm}$ and $570 \mu \mathrm{m}$.

A sample holder was designed to simultaneously oxidize four $12 \mathrm{~cm}$-long samples (see Fig. 1). Claddings have been oxidized in air-laboratory at $470{ }^{\circ} \mathrm{C}$, in a horizontal tubular furnace. Moreover, the tubes were equipped with end-caps to avoid internal oxidation. The end-caps were not fully gas-tight. An inner layer formed at the inner diameter but with a much thinner thickness (75\%) than the outer oxide layer.

Outer oxide layer thickness of $10,30,60,80$ and $100 \mu \mathrm{m}$ were obtained with exposure times of, respectively, 13, 45, 88, 128 and 151 days. Each sample remained in the furnace until the targeted oxide layer thickness was reached. After each high temperature period, the outer zirconia layer thickness was measured using a permascope, which has been calibrated by the supplier. Oxidized claddings were then cut into $1 \mathrm{~cm}$-long rings with an horizontal diamond wire saw, avoiding the degradation of the oxide layer thanks to the low pressure applied by the wire to the ring.

In order to evaluate the influence of inner and outer oxide scales on the load-displacement record, some rings with a $60 \mu \mathrm{m}$ thick outer oxide layer were fully or partly removed by polishing. A manual milling machine was used. This process enables complete removal of the oxide to the metal-oxide interface. Few oxide patches with thickness never exceeding $10 \mu \mathrm{m}$ sometimes remained adherent to the sample.

\subsection{Ring compression test}

An INSTRON 5566 testing machine, equipped with a $5 \mathrm{kN}$ load cell, was used to perform RCT at two temperatures $\left(20^{\circ} \mathrm{C}\right.$ and $350^{\circ} \mathrm{C}$ ). An INSTRON 3119 furnace was used for the $350^{\circ} \mathrm{C}$ tests.

Table 1

Chemical compositions of the studied alloys (wt\%).

\begin{tabular}{llllll}
\hline & $\mathrm{Sn}$ & $\mathrm{Fe}$ & $\mathrm{Cr}$ & $\mathrm{O}$ & $\mathrm{Zr}$ \\
\hline RXA Zy-4 & 1.29 & 0.21 & 0.10 & 0.128 & Base \\
SRA Zy-4 & 1.32 & 0.21 & 0.11 & 0.141 & Base \\
\hline
\end{tabular}



Fig. 1. Sample holder with four $12 \mathrm{~cm}$-long oxidized claddings.
Axial length of the ring samples, mechanically tested in this study, is $9.8 \mathrm{~mm} \pm 0.1 \mathrm{~mm}$. Only displacement-controlled tests were performed with a $1 \mathrm{~mm} / \mathrm{min}$ load-line displacement rate. The maximum load-line displacement must not exceed $8 \mathrm{~mm}$ in order to avoid excessive loads in the device. Actually, a large increase of sample stiffness during the last steps of sample deformation is expected. This elastic-stiffness increase is associated with two phenomena: a decrease of the radius of curvature at equatorial location, potentially followed by a contact between upper and lower internal faces of the ring. Compression strength and load-line displacement are recorded continuously.

\subsection{Scanning electron microscopy and optical observations}

Cross-section analysis was performed on specimens embedded in a resin and carefully prepared with an automatic grinding and polishing machine. Embedded samples were also observed either by a LEICA optical microscope, or by SX100 electron microprobe in the backscattered electron mode at low acceleration voltage $(20 \mathrm{kV})$. The observations were performed at the ring mid-section.

\section{Results}

\subsection{Preliminary}

Equatorial $\left(\theta=0^{\circ}\right.$ or $\left.\theta=180^{\circ}\right)$ and polar $\left(\theta=90^{\circ}\right.$ or $\left.\theta=270^{\circ}\right)$ locations of the ring are of particular importance for test interpretation and modeling (see Fig. 2).

\subsection{As-received Zircaloy-4}

The two materials (SRA and RXA) were first tested, in the as-received bare state, at $20^{\circ} \mathrm{C}$ and $350{ }^{\circ} \mathrm{C}$. The load-displacement curves of the compression tests are shown in Fig. 3. The ring first deforms from circular to elliptical shape. The contact surface is quite regular and planar. Then, for greater load-line displacements, it deforms into a peanut-shape (see Fig. 4). As a consequence, the contact surface divides into two contact bands symmetrically located on both side of the direction loading. This behavior has already been observed on aluminum rings [4].

For tests performed at room temperature, at large displacement (at about $5.5 \mathrm{~mm}$ for SRA Zy-4 and at $7.5 \mathrm{~mm}$ for RXA Zy-4) a significant stiffness decrease occurs. Optical examinations confirmed that this event is related to the failure of the cladding at the ring equatorial location where strains are important. As an example, an optical micrograph of RXA Zy-4 ring (see Fig. 4) exhibits large cracks at the equatorial azimuths. These cracks initiate at the outer surface of the ring and propagate toward the inner surface, and the crack depth is about half of the cladding thickness.

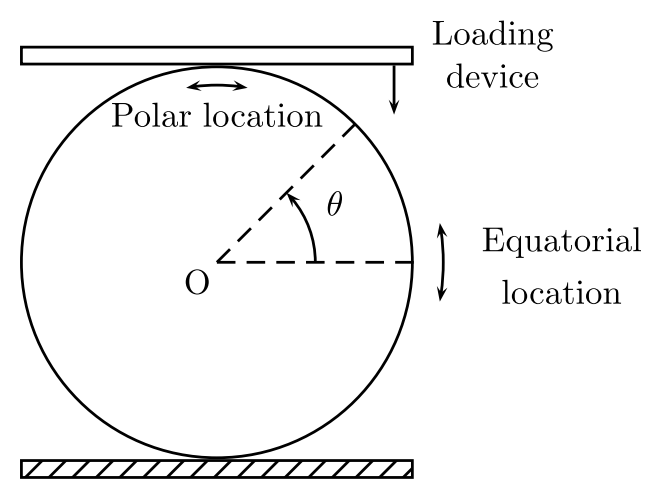

Fig. 2. Definition of equatorial and polar ring locations (axial direction view). 




Fig. 3. Load-displacement curves of Zircaloy-4 RCT at $20^{\circ} \mathrm{C}$ and $350{ }^{\circ} \mathrm{C}$.

The cladding failure was though never observed for ring compression tests performed at $350^{\circ} \mathrm{C}$. The maximum strain reached at ring equatorial azimuth remains lower than the failure strain.

\subsection{Oxidized Zircaloy-4}

RXA and SRA Zy-4 samples with different oxide layer thickness were submitted to RCT at room temperature. In this paper, only results for a set of five experiments performed on SRA rings covered by a $60 \mu \mathrm{m}$ thick outer oxide layer will be discussed. The difference between these five rings is the oxide grinding: inner and outer oxide were either partly or fully removed on specimens, as described in Table 2.

The load-displacement curves for these tests are shown in Fig. 5.

Similar load-displacement curves are observed for specimens with different oxide layer thickness. The typical mechanical response of an oxidized ring submitted to RCT is characterized by three losses of load carrying capacity events, as schematized in Fig. 6:

1. The greatest loss of load carrying capacity appears at load-line displacement comprised between $4.5 \mathrm{~mm}$ and $5 \mathrm{~mm}$. Indeed, this event corresponds to the cladding failure at both equatorial azimuth as already observed on non oxidized rings. All tested rings failed for quite similar strain level (see Fig. 5), except the specimen SR60\#1, which was likely not far from crack initiation. After this failure, two halves rings remain. The post failure behavior will not be further considered.

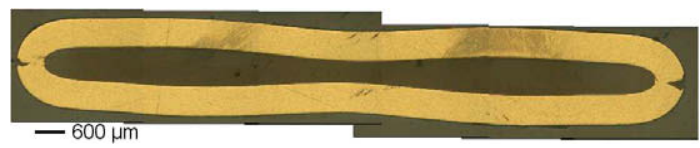

Fig. 4. Peanut-shape of a RXA Zy-4 ring after RCT at $20^{\circ} \mathrm{C}$. Optical micrograph on cross-section in the ring mid-section.

Table 2

Details on the oxide polishing of rings covered by a $60 \mu \mathrm{m}$ thick outer oxide layer.

\begin{tabular}{lll}
\hline Specimen & Removed & \\
\cline { 2 - 3 } & Outer oxide layer & Inner oxide layer \\
\hline SR60\#1 & None & None \\
SR60\#2 & None & Totally removed \\
SR60\#3 & $\theta=90^{\circ} \pm 20^{\circ}$ & Totally removed \\
SR60\#4 & Totally removed & Totally removed \\
SR60\#5 & $\theta=0^{\circ} \pm 45^{\circ}$ & Totally removed \\
\hline
\end{tabular}

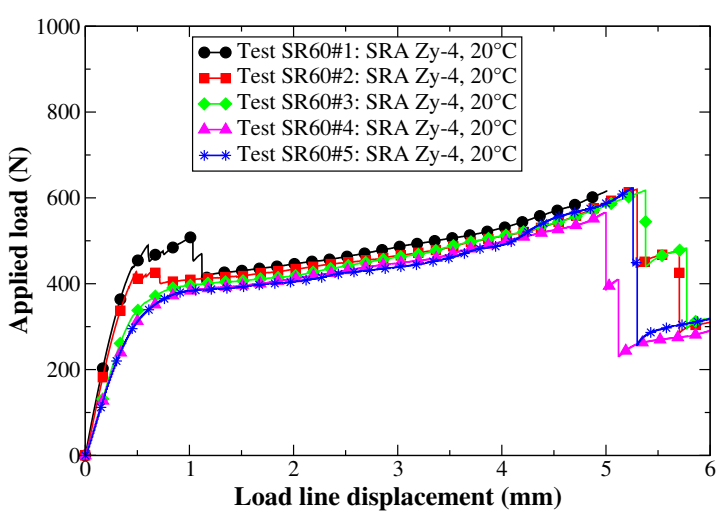

Fig. 5. Load-displacement curves for oxidized SRA Zy-4 ring compression test at room temperature.

2. Another event appears at load-line displacement close to $0.75 \mathrm{~mm}$. This event is related to buckling failure of the inner oxide layer at the equatorial azimuths (see Fig. 7). Buckling failures at opposite azimuths are not simultaneous, thus two similar events are observed at very close displacement values. To confirm this assessment, specimen SR60\#2 was polished over its entire inner surface to remove inner oxide layer. No loss of load carrying capacity is also observed for similar load-line displacement. Influence of the inner oxide layer on the mechanical response of an oxidized ring submitted to compression test is thus evidenced.

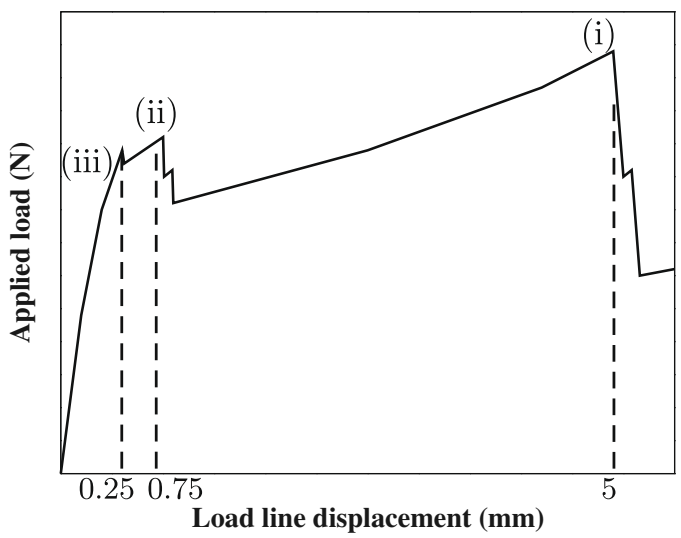

Fig. 6. Typical mechanical load-displacement curve of an oxidized ring compression test. Mentioned values concern results of RCT performed on ring covered by a $60 \mu \mathrm{m}$ thick outer oxide layer.

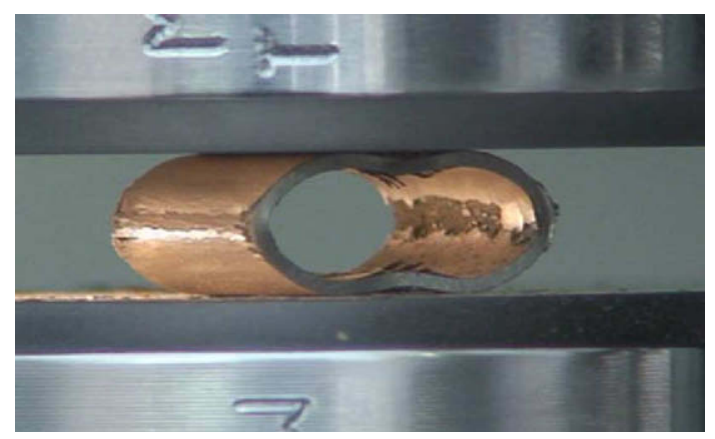

Fig. 7. Picture of an oxidized ring during RCT. 
3. A small failure-event is observed at a displacement corresponding approximately to the beginning of plasticity, when the loadline displacement reaches about $0.25 \mathrm{~mm}$. Visual examination during the test enables to conclude that the small loss of load carrying capacity is the consequence of oxide cracking under both device-specimen contact surfaces (at polar locations). Pole cracking is followed by the spallation of very small oxide fragments at these regions. This is confirmed by the test on sample SR60\#3, for which outer oxide has been removed at both poles.

As a conclusion, inner and outer oxide layers significantly contribute to increase the sample stiffness, until they fail. After the oxide cracking events, the mechanical behavior is the same for all rings and it is similar to the response of the base metal without any influence of oxide layers (SR60\#4).

Comparison between SR60\#4 and SR60\#5 tests (see Fig. 5) highlights that a large region of outer oxide layer has no main influence on the mechanical response of the ring. It also means that only zirconia layer submitted to compressive stresses improves significantly the strength of oxidized samples during RCT.

At locations corresponding to tensile stresses, oxide degradation can be evidenced. In particular, axially oriented oxide strips separated by axial-radial cracks are observed close to equatorial locations on the outer oxide layer (see Fig. 7).

\subsection{Oxide layer damage during $R C T$}

Figs. 7 and 8 evidence undoubtedly inner oxide spallation at equatorial azimuth: the oxide cracking is created by tensile strains

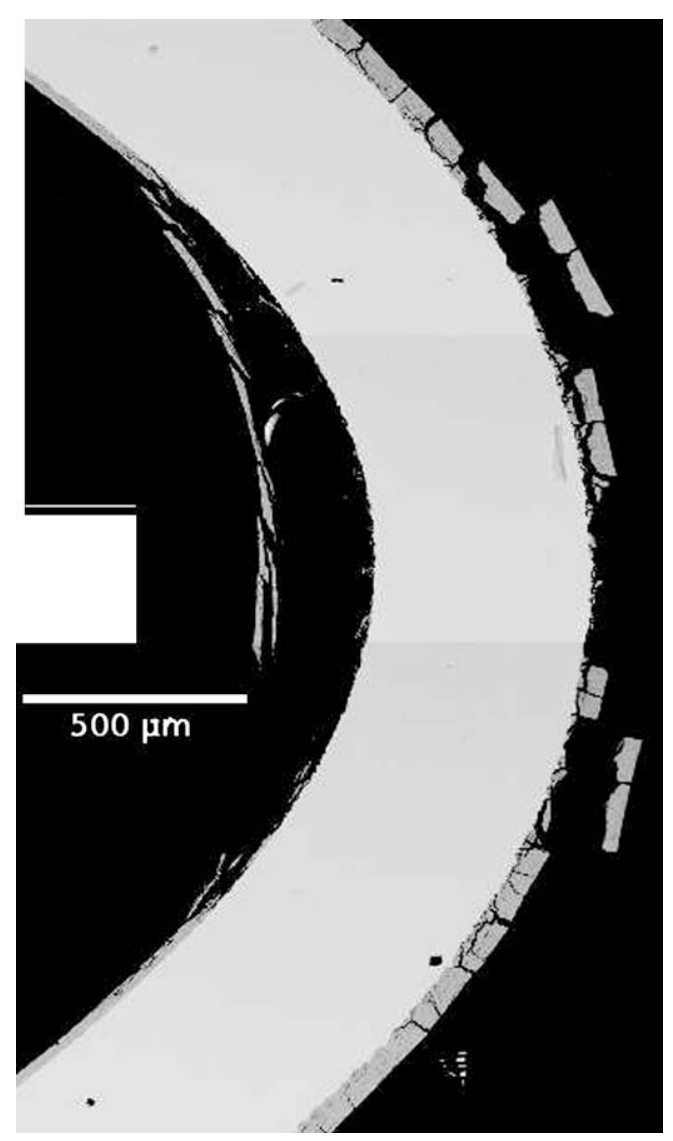

Fig. 8. SEM micrographs after RCT, on cross-section in the ring mid-section, in backscattered electron mode of RXA Zircaloy- 4 samples oxidized in air at $470{ }^{\circ} \mathrm{C}$. The outer oxide layer is $60 \mu \mathrm{m}$ thick. The corresponding load-line displacement is $5 \mathrm{~mm}$. on the external skin and inner-side oxide bucking induced by compressive stresses is observed. On the outer surface, many oxide fragments are spalled or close to spallation. The above-mentioned zirconia layer cracking at outer locations is related to the high strain level locally reached at the end of RCT [6]. On regions far from the equatorial azimuth, oxide damages are much more limited since most of the radial cracks do not fully propagate up to the metal-oxide interface. Ring compression tests appear to be particularly interesting to study oxide cracking and spallation under mechanical load. This study is underway and will not be further detailed here.

\section{Modeling}

\subsection{Two-dimensional plane strain conditions}

Three-dimensional finite element method (FEM) simulations of the ring compression were runned with the Cast3m software [10]. Only $1 / 8$ th of the ring is meshed because of the symmetries. The loading device is modeled by meshing a thick sheet. 22500 8-nodes cubic finite elements with linear interpolation were used for the ring meshing (see Fig. 9).

For this study, the ring mechanical behavior is assumed to be isotropic elastic-plastic. The isotropic strain-hardening tensile law is modelled using a power-law: $\sigma=K .\left(\varepsilon_{p}\right)^{n}$, where $\sigma$ and $\varepsilon_{p}$ are the equivalent Von-Mises stress and the corresponding equivalent plastic strain respectively and, $K$ and $n$ are two material parameters to be identified.

The loading device stiffness is known to be significantly higher than the sample stiffness. It is anyway assumed to be an elastic body with a large Young modulus, but material properties for loading device are expected to have a negligible influence on stress and strain distribution in the ring.

The mechanical properties of the modeled system are specified in Table 3.

The contact between the ring and the loading device is assumed to be perfect, without friction. A displacement-controlled loading is applied to the loading device. Large displacements and large strains are assumed in the calculation. No resmeshing of the model is done between calculation steps.

For a $1 \mathrm{~cm}$-long ring, the calculated axial strain is quite negligible compared to the hoop strain. The three-dimensional strain distribution in the ring is close to plane strain conditions. A criterion is introduced to check more accurately the strain state in the mod-

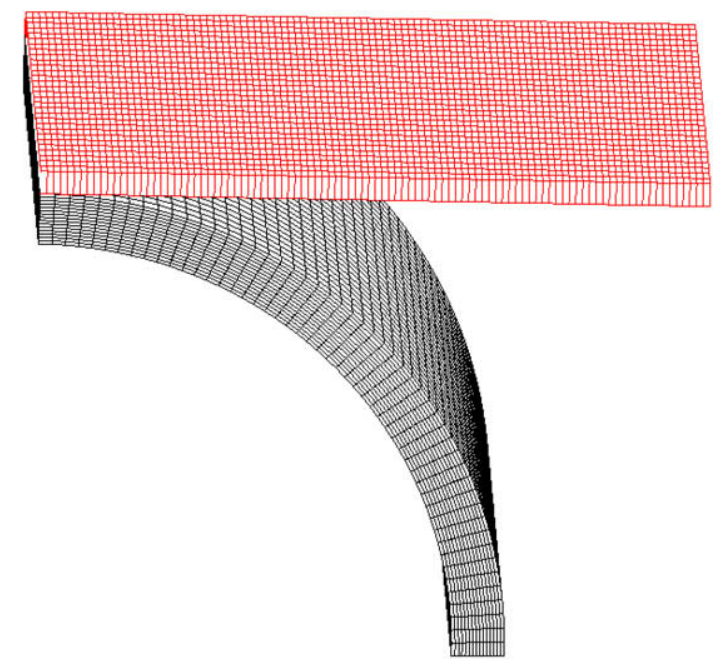

Fig. 9. Three-dimensional finite element method mesh. 
Table 3

Mechanical properties of the ring and the loading device.

\begin{tabular}{lll}
\hline Series & Series Ring & Series load. dev. \\
\hline Young modulus (MPa) & 98000 & 500000 \\
Poisson ratio & 0.325 & 0.325 \\
$K$ & 615 & \\
$n$ & 0.03 & \\
\hline
\end{tabular}

eled sample. Assuming here that $\varepsilon$ is the strain tensor expressed in the polar coordinates system linked to the ring, $\varepsilon_{P S}$ is the projection of this tensor to the plane strain components,

$\varepsilon_{P S}=\left(\begin{array}{ccc}\varepsilon_{r r} & \varepsilon_{r \theta} & 0 \\ \varepsilon_{r \theta} & \varepsilon_{\theta \theta} & 0 \\ 0 & 0 & 0\end{array}\right)$.

The following criterion is defined in order to evaluate the relative error between actual strain state and plane strain state:

$\eta=\frac{\left\|\varepsilon-\varepsilon_{P S}\right\|}{\|\varepsilon\|}$

The smaller $\eta$ is, the closer to plane strain state the strain distribution is. The evolution of $\eta$ is calculated at each node of the mesh (see Fig. 10). It can also be plotted along the axial direction at equatorial location (see Fig. 11). Considering a load-line displacement equal to $7 \mathrm{~mm}, \eta$ remains smaller than 0.05 over a $7 \mathrm{~mm}$ distance. It means that the plane strain state assumption is valid with a $5 \%$ accuracy along about $70 \%$ of the ring length. Remaining $30 \%$ is thus affected by three-dimensional edge effect.

Moreover, this conclusion remains valid whatever the load-line displacement is (see Fig. 12). The ring length which is near plane strain is slowly increasing with loading. This point is really interesting in particular for ring submitted to high strains. At equatorial azimuth and during the test, the strain levels are close to plane strain conditions in a region which extends on at least over $2 / 3$ of the axial length of the ring.

Consequently, the two-dimensional plane strain is an approximation of the three-dimensional real behavior (see Fig. 13). The computation time is thus strongly reduced.

\subsection{As-received Zircaloy-4}

Two-dimensional plane strain FEM numerical calculations are performed in order to identify the mechanical properties of the cladding (see Fig. 14). Fresh Zircaloy-4 anisotropic model is not perfectly known in the entire range of strains and strain rates. As a consequence, authors have chosen to model Zircaloy- 4 behavior

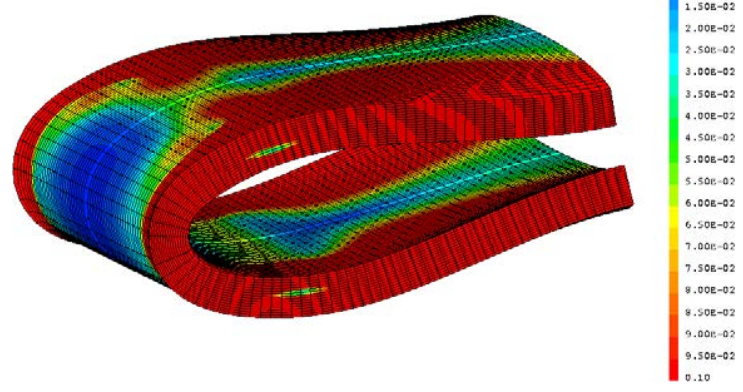

Fig. 10. Evolution of the calculated plane strain criterion for a $7 \mathrm{~mm}$ load-line displacement. $\eta$ is calculated at any nodes of the mesh. Only half of the ring is shown.

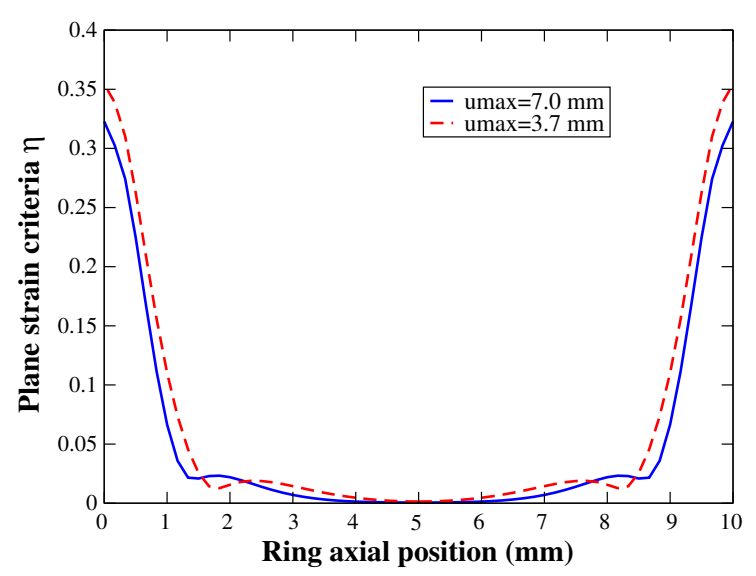

Fig. 11. Evolution of the calculated plane strain criterion at the equatorial azimuth versus the axial position of the ring for two maximum load-line displacements.

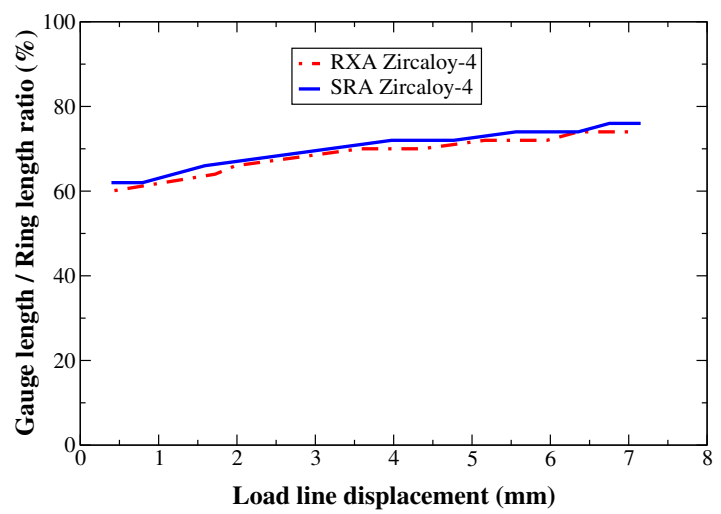

Fig. 12. Evolution of the axial length of the equatorial location, where the strain are close to plane strain conditions (with a 5\% accuracy), versus the load-line displacement.

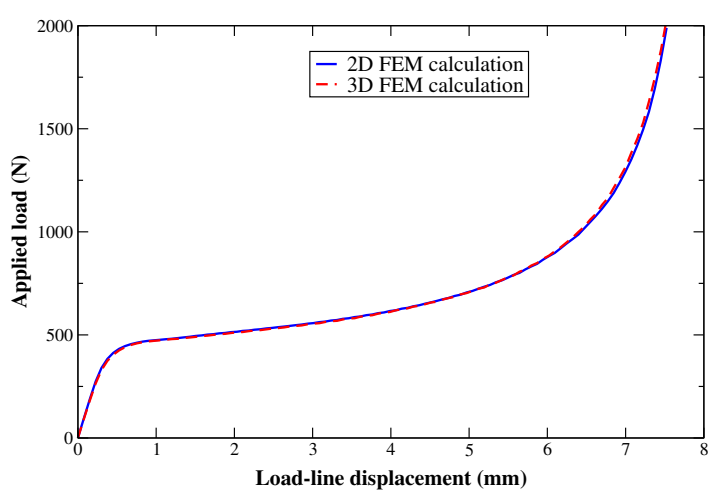

Fig. 13. Comparison of the calculated applied load versus load-line displacement for 3D and 2D plane strain FEM simulations.

with an isotropic model. A better characterization of the orthotropic behavior of the cladding would certainly contribute to a better assessment of the cladding mechanical properties. Cladding plasticity is assumed to follow a power-law stress-strain curve. The objective is here to determine these two material parameters $K$ and $n$ by fitting experimental and calculated load-displacement curves. When identified, the true stress-strain curve can be used to simulate a more standard test and then to derive usual engineering parameters such as the ultimate tensile strength (UTS), the $0.2 \%$ Yield Stress (YSO.2) and the uniform elongation (UE). 


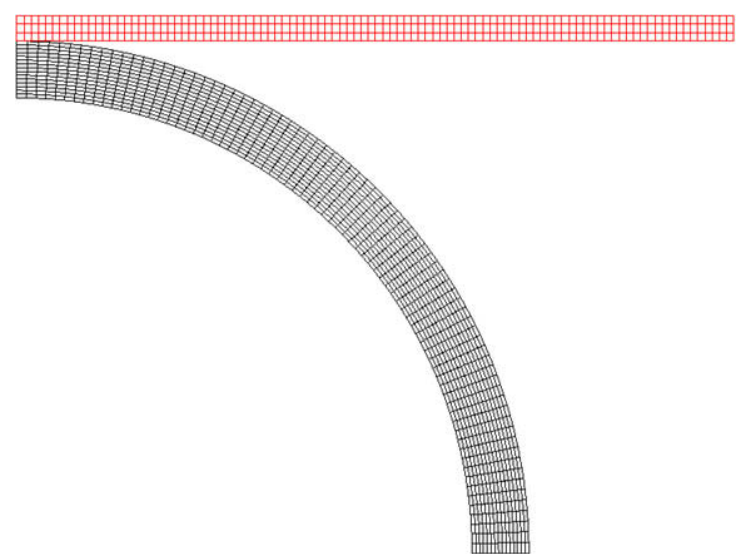

Fig. 14. Two-dimensional finite element mesh.

The load-line displacement behavior is assumed to be elastic (Young modulus $=500000 \mathrm{MPa}$, Poisson ratio $=0.325$ ).

Previous works $[3,5]$ have shown that it is possible to assess the UTS value, assuming elastic perfectly plastic material properties, with the following correlation:

$U T S=\frac{\alpha P_{0} R_{0}}{t_{0}^{2} l}$,

where $\alpha$ equals $\frac{\sqrt{3}}{2}$ for rings (longer than one diameter), $R_{0}, t_{0}, l$ are respectively the initial outer radius, the tube thickness and the length of the ring. $P_{0}$ is the initial collapse load, which can be obtained by the intersection of the extrapolation of elastic and plastic parts of the load-displacement curve (see Fig. 15). As a conclusion, the UTS is geometrically derived from load-displacement curve.

Numerical results are consistent with the experimental ones (see Fig. 16) and 0.2\% Yield Stress and Uniform Elongation are thus assessed by identification. Results are shown in Table 4 .

Another mechanical parameter, the failure strain, can also be obtained using numerical simulations of the RCT. The great loss of load carrying capacity observed for the two materials submitted to RCT at room temperature is a consequence of the cladding failure at equatorial azimuth. The crack initiation location during RCT on ductile material is always at the middle of the specimen at equator locations. Up to now, this has been systematically observed for all RCTs performed in the laboratory on ductile materials. The failure strain was derived by calculating the hoop strain in the middle of the equatorial line at a displacement corresponding to the loss of load carrying capacity. Results are summarized in Ta-

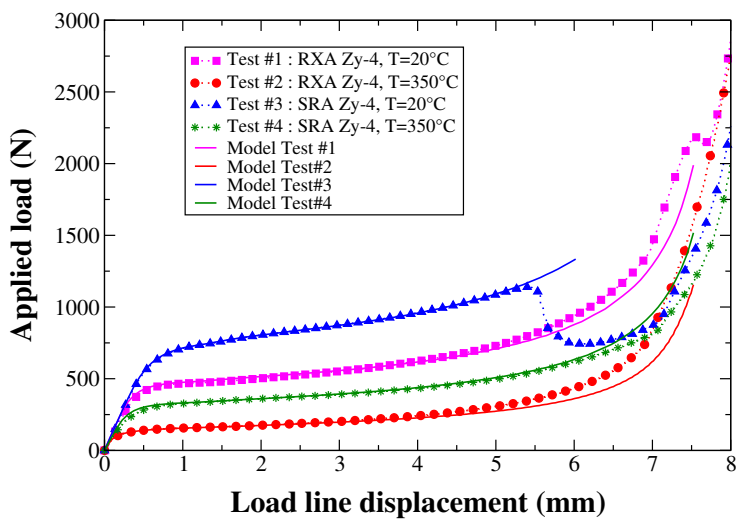

Fig. 16. Load-displacement curves of ring submitted to RCT.

Table 4

Mechanical properties identified on RCT of as-received Zircaloy-4.

\begin{tabular}{llllll}
\hline $\mathrm{T}\left({ }^{\circ} \mathrm{C}\right)$ & \multicolumn{2}{l}{$\mathrm{RXA} Z \mathrm{Zy}-4$} & & \multicolumn{2}{c}{ SRA Zy-4 } \\
\cline { 2 - 3 } & 20 & & & 20 & 350 \\
\hline Young modulus (MPa) & 98830 & 79360 & & 98830 & 79360 \\
Poisson ratio & 0.325 & 0.325 & & 0.325 & 0.325 \\
$K(\mathrm{MPa})$ & 640 & 312 & & 1040 & 475 \\
$n$ & 0.03 & 0.14 & & 0.03 & 0.047 \\
YSO.2 $(\mathrm{MPa})$ & 530 & 130 & & 855 & 350 \\
UTS (MPa) & 560 & 205 & & 905 & 390 \\
UE (\%) & 3 & 14 & & 3 & 4,7 \\
Failure strain (\%) & 42 & $\mathrm{NA}^{*}$ & & 26 & $\mathrm{NA}^{*}$ \\
\hline
\end{tabular}

* NA means not available from RCT without failure

ble 4. For example, concerning SRA Zircaloy-4 tests performed at room temperature, failure is obtained at $5.5 \mathrm{~mm}$-load-line displacement (see Fig. 16) and the corresponding plastic failure strain is $26 \%$ (see Fig. 17).

FEM calculations enable hoop strain rate calculation at the point of the ring where failure is expected (middle of equatorial line). The assessment of strain rate is obtained in a two step process: the derivative of the strain versus displacement (see Fig. 18) is first calculated, and then multiplied by the load-line displacement rate. During tests with load-line displacement rate of $1 \mathrm{~mm} / \mathrm{min}$, the strain rate remains rather constant and close to $5 \times 10^{-4} \mathrm{~s}^{-1}$ before significant deformation of the ring. During the last steps of deformation, corresponding approximately to situation with contact between opposite sides of the inner-diameter of the ring, a sudden

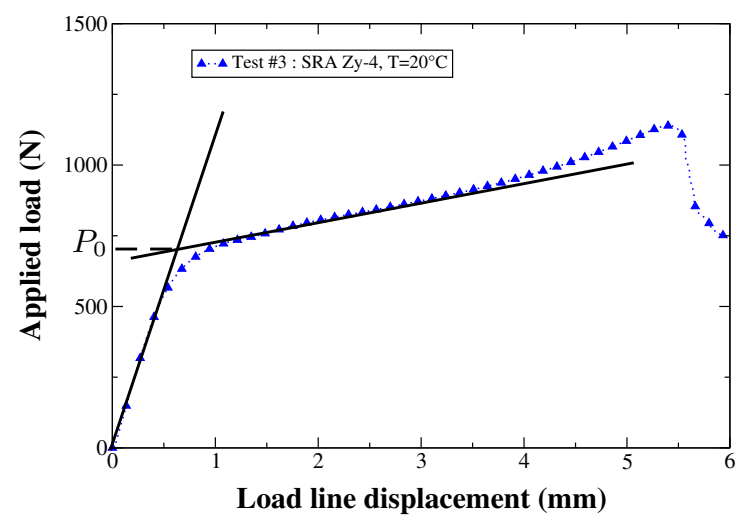

Fig. 15. Definition of the initial collapse load, as mentioned in [3,5].

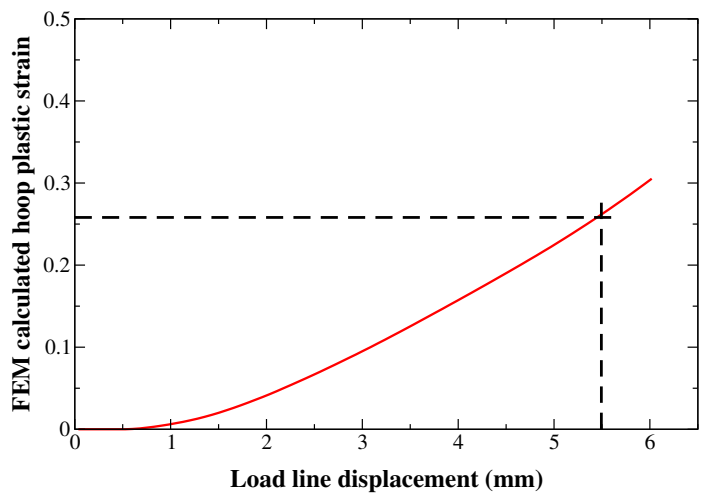

Fig. 17. Evolution of hoop plastic strain at the equatorial location during RCT for SRA Zy-4 at room temperature. 


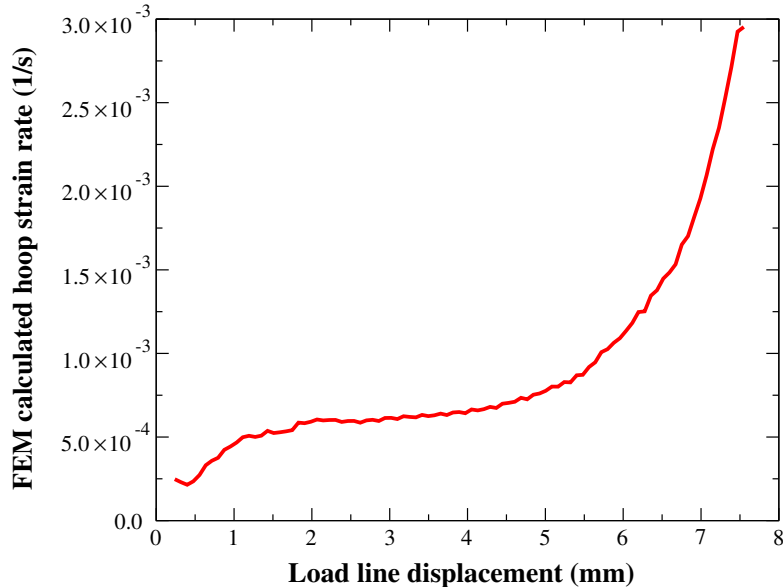

Fig. 18. Evolution of the hoop strain rate at the equatorial location during RCT.

increase of strain-rate is calculated. The strain rate plateau can be easily adjusted to any value by choosing the relevant load-line displacement rate. Apart from this study, an experimental sensitivity study of the strain rate influence has been performed on non oxidized cladding (from $5 \times 10^{-4} \mathrm{~s}^{-1}$ to $5 \times 10^{-1} \mathrm{~s}^{-1}$ ). Limited strain rate hardening is observed at room temperature.

\subsection{Oxidized Zircaloy-4}

Zirconia is assumed to follow an elastic behavior (Young modulus $=168000 \mathrm{MPa}$, Poisson ratio $=0.25$ ). The oxide coating is meshed using about ten 8-nodes elements with linear interpolation in the thickness direction.

Air oxidation and associated annealing at elevated temperature induces cladding microstructure change. Actually, SRA Zircaloy-4 reaches a fully recrystallized state after 10 days at $470{ }^{\circ} \mathrm{C}$ [11]. In other words, the mechanical strength of the originally SRA Zircaloy-4 slowly decreases and merges with RXA Zircaloy-4 mechanical properties. Mechanical testing was performed at room temperature after oxidation. Ring material properties were thus assumed to follow the flow rule of recrystallized Zircaloy-4, i.e. with the following power-law parameters: $K=640 \mathrm{MPa}$ and $n=0.03$.

Besides, part of the base metal is consumed during the oxidation treatment. The end-of-oxidation cladding thickness $e_{c l a d}$ is thus slightly reduced and it can be quite accurately predicted by the following correlation [12]:

$e_{\text {clad }}=e_{\text {clad }}^{0}-0.636\left(e_{\text {oxide }}^{\text {out }}+e_{\text {oxide }}^{\text {in }}\right)$,

where $e_{\text {clad }}^{0}$ (here $\left.570 \mu \mathrm{m}\right), e_{\text {oxide }}^{\text {out }}, e_{\text {oxide }}^{\text {in }}$ are the initial cladding thickness, the outer and inner oxide layer thickness, respectively.

As previously shown, oxide regions in tensile state have negligible mechanical strength and poorly contribute to the overall mechanical response during the test. Numerical simulations highlighted that, as soon as RCT begins, the metal-oxide interface is subjected to tensile state in two regions enclosed by the outer surface of the ring from $0^{\circ}$ (equatorial azimuth) of $45^{\circ}$ and by the inner surface from $45^{\circ}$ to $90^{\circ}$. These regions are thus not included in the finite element model.

Experimental load-displacement response of an oxidized ring is characterized by three main events (outer oxide layer cracking at polar locations then inner oxide layer cracking at equatorial location then cladding failure) related to extended and well-defined areas with damaged oxide. Three corresponding finite element models have thus been developed. The evolution of damage within the oxide is thus modeled once for all with each of the three models.
- For model \#1, outer and inner oxide layers are meshed (see Fig. 19(a)).

- In model \#2, only the inner oxide layer is modelled (see Fig. 19(b)).

- No zirconia layer is meshed in model \#3. This last calculation is thus similar to the one previously described for as-received Zircaloy-4, with reduced cladding thickness (see Fig. 19(c)).

Numerical results consistently describe the main steps of the oxide damage that were observed during experiments (see Fig. 20). Actually, model \#1 is valid at the very beginning of the test until the outer oxide layer fails near the contact area with the loading device. After this first event, model \#2 is expected to reproduce the load evolution until the inner oxide layer failure. Experimental results exhibit higher stiffness than numerical ones. It could be explained by the fact that the polar outer oxide cracking does not occur exactly at $45^{\circ}$. Actually, apart from regions in close vicinity with the loading-device-sample contact surface, most of the outer oxide layer remains undamaged. Concerning model \#3, numerical results are consistent with experimental data. As a conclusion, FEM calculations confirm the different oxide layer cracking mechanisms identified experimentally.
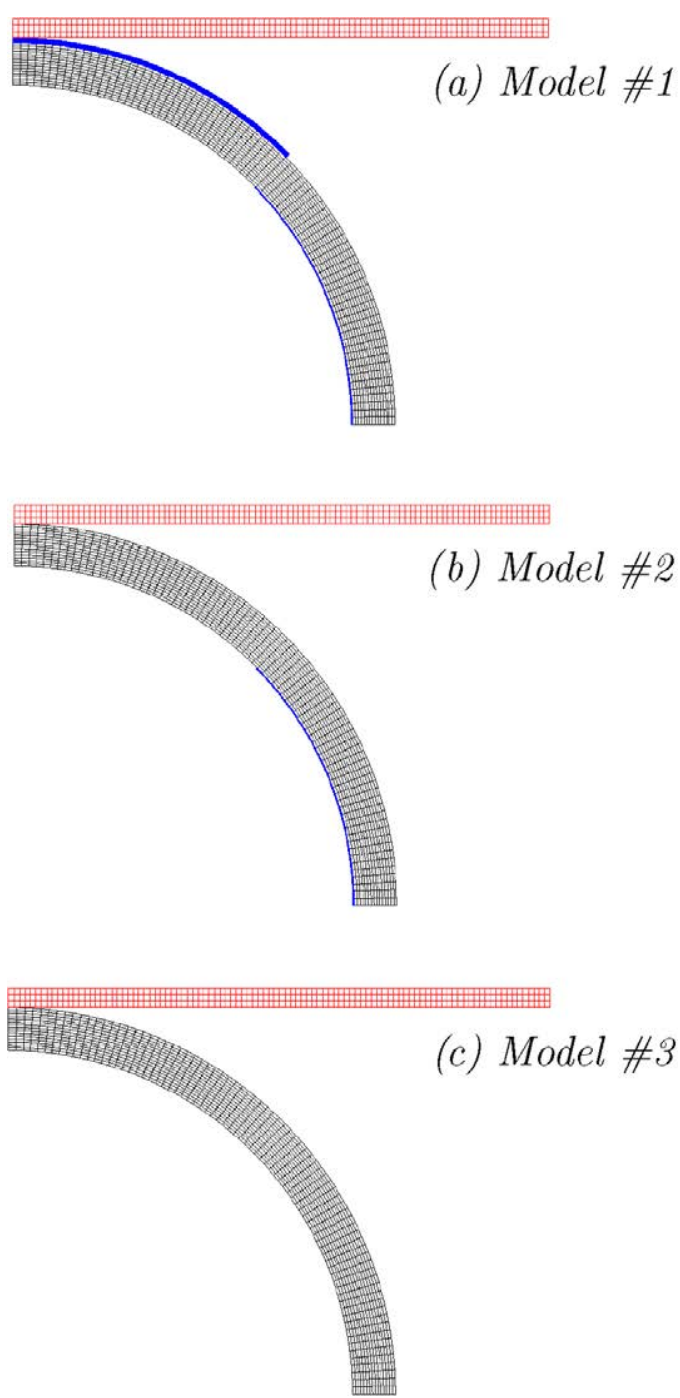

Fig. 19. FEM models for Zircaloy-4 ring covered by a $60 \mu \mathrm{m}$ and $15 \mu \mathrm{m}$ thick outer and inner oxide layer, respectively. 


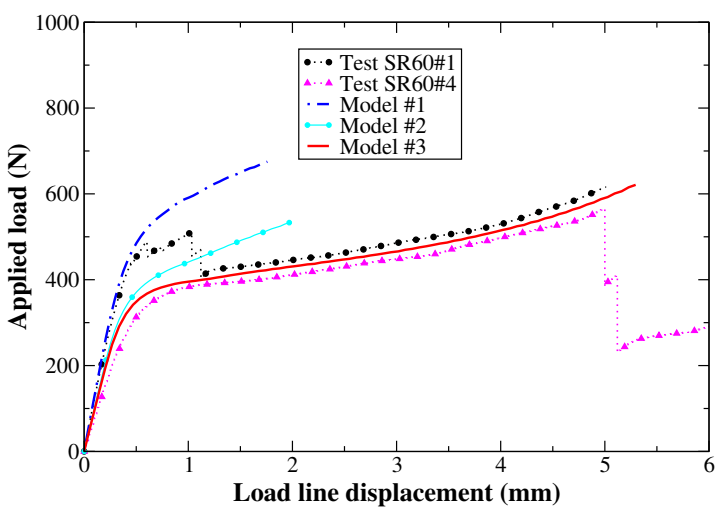

Fig. 20. Experimental and calculated load-displacement curves of Zircaloy-4 RCT.

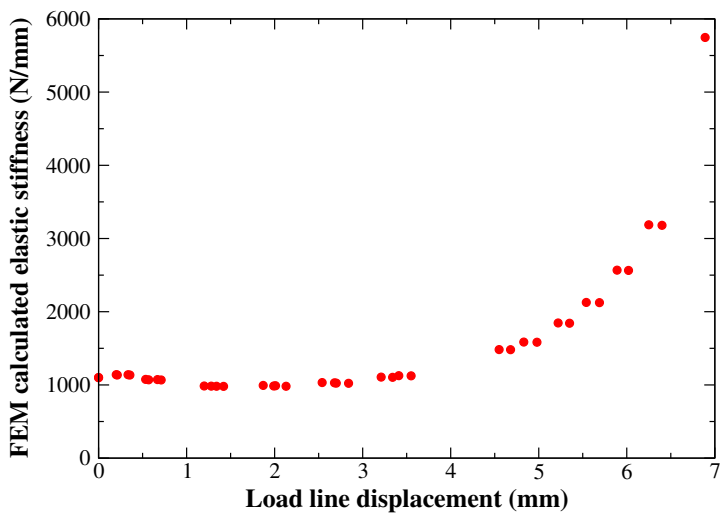

Fig. 21. Evolution of FEM calculated ring elastic-stiffness versus load-line displacement.

\section{Discussion}

Failure strain values deduced from RCT at room temperature are consistent with total elongation values obtained using ring tensile tests recently performed by CEA in the frame of the PROMETRA program. The typical geometry of hoop tensile tests is described in [13]. Total elongation obtained within the PROMETRA program with similar material and testing conditions was about $16 \%$. This result is not so far from the $26 \%$ failure strain deduced from RCT in the present study. For highly ductile material, RCT-derived failure strains appeared to be significantly higher than expected. It is thus not possible to conclude that the obtained failure strain is equal to the total elongation. The hoop tensile samples of the PROMETRA program have a well-defined gage section whereas RCT samples has a localized and changing gage section. This might explain the difference between the values deduced from both mentioned tests. Regarding the other material parameters (Ultimate Tensile Strength, $0.2 \%$ Yield Stress and Uniform Elongation), values deduced from RCT are consistent with values deduced from PROMETRA ring tensile tests performed on similar Zircaloy-4, which are still unpublished.

Parametric calculations have been performed in order to assess the influence of friction between the Zircaloy-4 ring and the loading device. The friction coefficient was varied from 0 to 1 and had negligible influence on the mechanical response of the test. This can be linked to a rolling-like contact on a localized area during the test, as mentioned in $[4,6]$.

In LOCA studies, claddings are much more embrittled than the one considered in the present study (brittle oxygen stabilized $\alpha$ -
$\operatorname{Zr}(\mathrm{O})$ layer...). In order to avoid or delay the LOCA-specimen failure at polar location, a slot is usually machined in the loading device [8]. This slot also contributes to maintain the sample after pole cracking. But, for the ductile materials considered in the present study, failure first happens at equatorial location and was never observed at polar location. As a conclusion, for ductile materials, there is no need for a slot in the loading device.

FEM calculations, with a set of loading and unloading during the test at several steps, have been performed to evaluate the evolution of the ring elastic-stiffness during the test (see Fig. 21). The ring elastic-stiffness remains quite constant for the first $4 \mathrm{~mm}$ load-line displacement, then a large increase is predicted. Thus plastic displacement can be easily calculated for the beginning of the test taking into account initial elastic-stiffness. However, it needs to be calculated carefully for load-line displacements exceeding $4 \mathrm{~mm}$.

\section{Conclusion}

Ring compression test is an interesting test method to determine some useful mechanical properties of cladding tubes. Its reduced machining cost and the low material consumption give to the test a real potential. Moreover, the test has no sensitivity to friction between the loading device and the sample, whereas the friction remains a complex and influent parameter when considering ring tensile test loaded with inserted mandrels.

An inverse method, based on finite elements calculations, can be run. The corresponding results are compared to the experimental ones to determine parameters, such as the Ultimate Tensile Strength, the $0.2 \%$ Yield Stress and the Uniform Elongation. This study showed that a $10 \mathrm{~mm}$ long ring sample provides reasonably plane strain conditions. With more than $10 \mathrm{~mm}$ long ring samples, a two-dimensional numerical simulations under plane strain assumption provides with sufficient accuracy a good mean to analyze load-displacement curves. It is also possible to assess the evolution of stress or strain at each point of the ring and at any applied displacement. However, one important drawback of such a test is that the material behavior is investigated simultaneously for tensile and compressive loading. Without complementary information, it is thus necessary to assume that material stress-strain curve is similar under tensile and compressive stress.

The RCT test has a great potential to analyze the mechanical behavior of irradiated claddings, which are usually embrittled by hydride rims at the outer diameter of the cladding. When performing a RCT on irradiated claddings, the first crack is expected at the outer diameter of the equatorial location. Actually, the inner diameter remains more ductile. The analysis of oxide influence during RCT shows that oxide layer has to be removed. Efforts are now underway to implement RCT test in hot cells at CEA.

Oxide spallation and cracking can be interestingly addressed using post-RCT metallographic examination. Further efforts are underway to describe locally the oxide degradation mechanisms under mechanical loading.

\section{Acknowledgement}

This work was partly supported by AREVA NP.

\section{References}

[1] V. Georgenthum et al., J. Nucl. Sci. Technol. 43 (2006) 1089.

[2] S.R. Reid, T. Yella Reddy, Int. J. Solids Struct. 14 (1978) 213.

[3] T. Yella Reddy, S.R. Reid, Nucl. Eng. Design 52 (1979) 257.

[4] T. Yella Reddy, S.R. Reid, Int. J. Solids Struct. 16 (1980) 545

[5] M. Nemat-Alla, Int. J. Mech. Sci. 45 (2003) 605. 
[6] M. Avalle, L. Goglio, J. Strains Anal. 32 (1997) 335.

[7] O. Calme et al., Compos. Sci. Technol. 65 (2005) 95.

[8] Y. Yan, et al., Report NUREG/CP-0185: LOCA Results for Advanced-Alloy and High-Burnup Zircaloy Cladding, 2003 Nuclear Safety Research, 20-22 October 2003, Washington DC.

[9] A. Stern et al., J. ASTM Int. (2008) 5.

[10] http://www-cast3m.cea.fr.
[11] P. Bouffioux, L. Legras, Effect of hydriding on the residual cold work recovery and creep of Zircaloy-4 cladding tubes, Light Water Reactor Fuel Performance, 10-13 April 2000, Park City.

[12] L. Robert-Bérat, Influence d'une couche de zircone sur le comportement mécanique des tubes en Zircaloy-4, PhD thesis Université Blaise Plascal, 2001.

[13] B. Cazalis et al., Nucl. Technol. 157 (2007) 215. 